\title{
CJLT

\section{Synthèse critique des connaissances sur l'écriture électronique à l'aide du blogue au primaire et au secondaire}

\section{A Critical Synthesis of the Knowledge Surrounding Electronic Writing Using Blogs at the Elementary and High School Levels}

Stéphane Allaire, Université du Québec à Chicoutimi

Pascale Thériault, Université du Québec à Chicoutimi

Evelyne Lalancette, Université du Québec à Chicoutimi

\section{Résumé}

Cet article présente une synthèse critique des connaissances qui s'inscrit dans la foulée des travaux du Comité d'experts sur l'apprentissage de l'écriture qui, en janvier 2008, a publié un rapport intitulé « Mieux soutenir le développement de la compétence à écrire ». La synthèse s'est concentrée sur les textes à caractère scientifique traitant de la pratique d'écriture électronique du blogue dans les classes du primaire et du secondaire. La période couverte s'étend de janvier 2004 à mai 2010. Les résultats indiquent que les contextes d'utilisation du blogue peuvent favoriser la motivation à écrire des élèves, qu'ils procurent une authenticité à la démarche d'écriture et qu'ils amènent les élèves à produire de nombreux écrits. Toutefois, la dimension qualitative de ces derniers a été peu abordée jusqu'à présent. En outre, à la lumière des écrits recensés, des considérations conceptuelles et méthodologiques sont proposées à titre de pistes de recherche éventuelles.

\section{Abstract}

This article offers a critical synthesis of the knowledge obtained in the wake of the work of the Comite d'experts sur l'apprentissage de l'écriture (Committee of experts on learning to write), which published a report entitled, « Mieux soutenir le développement de la compétence à écrire » (Better Support for the Development of Writing Skills) in January 2008. The synthesis focused on scientific texts that deal with the practice of electronic blog writing in elementary and high school classrooms. The period covered extends from January 2004 to May 2010. Results indicate that a blog's contexts of use can favour students' motivation to write, add authenticity to the writing process, and lead students to produce numerous texts. The qualitative aspect of these texts, however, has been little discussed until now. In light of the compiled texts, conceptual and methodological considerations are also proposed as potential avenues for future research. 


\section{Introduction}

Cette synthèse critique des connaissances s'inscrit dans la foulée des travaux du Comité d'experts sur l'apprentissage de l'écriture qui, en janvier 2008, a publié un rapport intitulé « Mieux soutenir le développement de la compétence à écrire ». La qualité du français faisant l'objet de nombreux questionnements au Québec, le Comité s'était vu confier le mandat de « déposer un avis et des recommandations sur l'apprentissage du français écrit au primaire et au secondaire » (Ministère de l'Éducation, du Loisir et du Sport [MELS], 2008, p. 1).

C'est dans cette optique que la Direction de la recherche, des statistiques et de l'information du MELS a sollicité une étude à propos de l'écriture électronique à l'aide du blogue dans les classes du primaire et du secondaire. L'objectif de cette étude était de procéder à une synthèse critique des connaissances. C'est ce dont nous rendons compte dans le présent texte. ${ }^{1}$

\section{Problématique}

La compétence à écrire est fondamentale puisque, avec la lecture, elle constitue le socle des autres apprentissages scolaires. Elle est aussi névralgique pour l'émancipation individuelle dans une société de plus en plus qualifiée « du savoir », comme en témoignent les orientations préconisées par des organismes tels le « Partenariat pour les habiletés du XXI ${ }^{\mathrm{e}}$ siècle ${ }^{2}{ }^{2} \mathrm{Il}$ est reconnu que l'écriture est une activité de construction de sens (Allington, 2010; Simard, 1995) complexe (Barré-De Miniac, 2000) qui s'orchestre autour de trois principaux processus : la planification, la mise en texte et la révision (Hayes, 1995 ; Hayes \& Flower, 1980). Afin qu'une telle activité soit porteuse et féconde pour celui qui y prend part, nombre d'auteurs insistent notamment sur l'importance d'y intervenir de façon stratégique (Coker \& Lewis, 2008) et de l'ancrer dans un contexte de communication authentique (Allington, 2010; Allington \& Cunningham, 1998; Atwell, 1998; Graves, 2003).

Que ce soit au primaire ou au secondaire, les domaines disciplinaires décrits dans le «Programme de formation de l'école québécoise » encouragent l'authenticité des contextes d'écriture. En plus du domaine des langues, la mathématique, la science et la technologie, les arts, l'éthique et l'univers social sont autant de lieux qui permettent aux élèves d'écrire avec l'intention de partager des idées, de réagir, d'informer, de questionner, de résoudre des problèmes, etc. Qu'il s'agisse d'écrire pour soi (clarifier sa pensée, conserver des traces, planifier, etc.) ou à un destinataire, les élèves sont amenés à mobiliser des stratégies d'écriture permettant de faire de leurs textes un tout pertinent et cohérent (Ministère de l'Éducation du Québec [MEQ], 2001; MELS, 2006). Qui plus est, ces différentes situations d'écriture peuvent être menées à l'aide d'outils, de médias et de supports variés, conférant alors une dimension électronique à l'écriture.

Les questionnements à propos du potentiel de l'ordinateur et de l'écriture électronique pour l'amélioration de l'enseignement et de l'apprentissage de l'écriture ne proviennent pas d'hier (Hawisher, 1989; Kaplan, 1991). Notamment, Daiute (1983, 1985) a montré le potentiel du

\footnotetext{
${ }^{1}$ Cette étude a pu être menée grâce à un financement obtenu du MELS dans le cadre d'un projet de recherche ciblé en écriture.

${ }^{2}$ Pour en savoir davantage, le lecteur est invité à consulter http://www.p21.org/
} 
traitement de texte pour l'augmentation du temps consacré à la révision des textes, une étape importante contribuant à en améliorer la qualité. Cochran-Smith, Paris et Kahn (1991) ont soutenu que l'utilisation de l'ordinateur pouvait engendrer des changements dans la façon dont on apprend à écrire. D'autres auteurs ont rendu compte de la façon dont les ressources informatiques peuvent contribuer au changement de la dynamique de classe en favorisant l'autonomie des élèves du fait que ces derniers ont accès plus aisément à des outils les soutenant dans leur démarche d'écriture (Dickinson, 1986; Herrmann, 1987; Snyder, 1993, 1994). Ces études et réflexions à propos de l'écriture électronique ont été menées à une époque où le World Wide Web, le protocole le plus utilisé de l'Internet, n'était pas accessible dans les écoles, voire n'existait pas.

La situation est toute autre depuis une quinzaine d'années, et particulièrement depuis 10 ans avec la croissance exponentielle des technologies du Web 2.0 (DiNucci, 1999; O’Reilly, 2004) (aussi appelées médias sociaux). Ces médias sociaux, dont le blogue fait partie, ont transformé Internet en un lieu d'expression et de communication, amenant ainsi de nouvelles opportunités en matière de pratiques d'écriture électronique. Cela marque un changement important par rapport au Web 1.0 qui est davantage orienté vers la consultation d'information. Cette croissance est telle qu'à titre d'exemple, en 2005 seulement, près de cinquante millions de blogues ont été créés («Dossier - Les bogues », 2005). Ces technologies ont gagné la ferveur du public, dont les jeunes. Pour plusieurs d'entre eux, les nouvelles pratiques d'écriture qui découlent de l'utilisation de tels outils font non seulement partie de leur quotidien, elles sont aussi au cœur de leurs routines communicationnelles (Roy, 2009).

Les jeunes appartenant à la génération des digital natives (Prensky, 2001) sont familiers avec le monde des technologies de l'information et de la communication (TIC) (Roy, 2009). Ils en font une utilisation personnelle importante. Malgré l'évacuation récente des compétences transversales du « Programme de formation de l'école québécoise », dont celle concernant spécifiquement les TIC, il demeure que l'école a un rôle d'éducation important à jouer auprès des élèves en favorisant le développement de compétences permettant un usage pédagogique adéquat de ces outils. Les nouvelles perspectives d'apprentissage offertes par les TIC, l'accessibilité à une multitude de sources d'informations et de possibilités de collaboration ainsi que le nombre grandissant d'emplois exigeant l'utilisation des TIC - des emplois souvent centrés sur la création et l'innovation et qui sont dits de la « société du savoir » - sont autant d'arguments justifiant la pertinence de les intégrer aux activités scolaires. Or, l'étude du Roy (2009) indique que de tels outils sont encore bien peu exploités à l'école.

Pourtant, l'utilisation d'un outil tel le blogue peut contribuer à prendre conscience que l'écriture est une pratique sociale (Downes, 2006; Ward, 2004) et qu'elle dépasse largement la seule utilisation qu'on peut faire de l'écriture dans le cadre de travaux scolaires. Cela semble offrir un potentiel intéressant au regard du caractère authentique qui gagne à caractériser toute démarche d'écriture. D'autres auteurs (Alexander, 2006; Boyd, 2006) soutiennent que l'écriture électronique à l'aide d'un blogue permet d'inscrire le développement de la compétence à écrire à travers des orientations collaboratives qui souscrivent à des pratiques pédagogiques contemporaines fondées sur une perspective sociale de l'apprentissage (voir, par exemple, Greeno, 1998). À cet effet, l'accès à de nouveaux outils dits sociaux n'est possiblement pas étranger à l'émergence de nouvelles orientations dans le domaine de l'écriture. Par exemple, Hayes (2006) discute de l'adoption du cadre de référence de la théorie de l'activité (Engeström, 
1999), dont la dimension systémique peut permettre de saisir les relations en jeu dans l'activité d'écriture entre des éléments tels les caractéristiques des outils d'écriture utilisés, les scripteurs, leurs objectifs, la façon dont ils organisent la tâche, les conventions à respecter et la communauté à qui ils s'adressent.

Si l'utilisation du blogue est répandue dans le public et dans la vie personnelle des jeunes (Roy, 2009), cette pratique d'écriture électronique demeure néanmoins en émergence dans les classes du primaire et du secondaire, malgré l'engouement qu'elle peut susciter chez les jeunes. Il en va de même pour la recherche qui s'y est intéressée. En vue de faire le point sur l'état actuel des connaissances et d'identifier des pistes de recherche pertinentes, l'étude visait donc à documenter les recherches qui ont été effectuées ces dernières années sur cette modalité d'écriture $^{3}$ aux ordres primaire et secondaire. Dans un premier temps, nous explicitons la démarche utilisée pour effectuer la recension des écrits. Nous poursuivons en présentant les résultats des textes retenus, résultats dont l'organisation s'articule en quatre volets qui ont émergé de l'analyse. En troisième lieu, nous posons un regard critique sur les aspects conceptuels et méthodologiques des écrits recensés. Nous concluons avec des propositions d'implications pour les recherches ultérieures.

\section{Méthodologie}

Cette section explique la démarche méthodologique mise en œuvre dans le cadre de la synthèse critique des connaissances.

\section{Objectifs}

Les objectifs suivants ont été formulés :

a) identifier les écrits à caractère scientifique qui rendent compte d'utilisations du blogue en contexte scolaire, et principalement celles qui sont de l'ordre de l'apprentissage de l'écriture ;

b) procéder à une synthèse critique des écrits retenus dans une optique d'identification de pistes de recherche ultérieures.

\section{Démarche de la synthèse critique des connaissances}

La démarche nécessaire à la recherche de textes, et plus particulièrement celle liée aux ressources consultées, est expliquée ci-après. Les critères de sélection privilégiés pour le choix des textes sont également spécifiés.

\section{Procédure de recherche des textes}

Il a été convenu de procéder à la recherche de textes en considérant la période 2004-2010 (jusqu'en mai), inclusivement, cela afin d'accroitre le plus possible la pertinence des résultats en regard des orientations en recherche et des pratiques pédagogiques actuelles. À l'aide de nombreux mots-clés, la recherche de textes a été effectuée en considérant différentes ressources, dont majoritairement des bases de données reconnues en éducation.

\footnotetext{
${ }^{3}$ Nous précisons que les contextes d'utilisation des microblogues (par exemple Twitter) n'ont pas été considérés dans le cadre de cette étude.
} 
Les mots-clés présentés au Tableau 1 ont été utilisés pour la recherche de textes, qui s'est faite en anglais et en français. Une variété de combinaisons de mots-clés ont été effectuées à partir des opérateurs booléens.

Tableau 1: Mots-clés utilisés pour la recherche de textes

\begin{tabular}{|l|l|}
\hline Mots-clés de langue anglaise & Mots-clés de langue française \\
\hline Electronic publishing & Publication électronique \\
\hline Digital, electronic and online writing & Écriture électronique et numérique \\
\hline Social networks & Réseaux sociaux \\
\hline Writing, writing processses & Écriture, processus d'écriture \\
\hline ICT integration & Intégration des technologies \\
\hline Blog, weblog, blogging & Blogue, carnet, cybercarnet \\
\hline Hybrid learning environment & Environnement d'apprentissage hybride \\
\hline Digital and electronic environment & Environnement numérique \\
\hline Web 2.0 & Web 2.0 \\
\hline Digital media & Médias sociaux \\
\hline ICT affordances & Affordances des TIC \\
\hline $\begin{array}{l}\text { Primary school, secondary school, high } \\
\text { school }\end{array}$ & École primaire et école secondaire \\
\hline
\end{tabular}

Considérant que la recension se concentrait sur les écrits à caractère scientifique, les outils de recherche et les banques de données suivants ont été consultés :

- Google Scholar

- Education Resources Information Center (ERIC)

- Érudit

- Taylor \& Francis

- CBCA Education (ProQuest)

- Education Research Complete

Par souci d'exhaustivité, des recherches différentes ont été effectuées à plusieurs reprises par une même personne entre les mois de novembre 2009 et de mai 2010. En outre, certaines recherches ont été menées par une seconde personne à des fins de triangulation et de contre-vérification des résultats. 


\section{Procédure et critères de sélection des textes}

L'éventail des mots-clés utilisés a donné lieu à une recherche exhaustive qui a généré une quantité importante d'articles. Ultimement, ils ont été sélectionnés à partir de trois critères. Ainsi, pour être retenu, un texte devait :

- traiter de l'utilisation du blogue au primaire ou au secondaire ;

- posséder un caractère scientifique, c'est-à-dire une forme de problématisation, des objectifs et/ou des questions de recherche, et un cadre de référence ;

- rendre compte de résultats issus d'une démarche systématique de collecte de données empiriques menée dans le cadre d'une expérimentation.

De la centaine de textes identifiés au départ, une présélection a été effectuée sur la base de la lecture de leur résumé. Cette présélection a été menée principalement par la même personne, mais l'élimination de textes a été validée par deux autres personnes avant de les écarter de façon définitive. En outre, la bibliographie de certains textes qui étaient particulièrement en lien avec l'objet de la recension a aussi été consultée afin d'identifier des textes d'intérêt supplémentaires qui pouvaient ne pas avoir été répertoriés par les recherches dans les banques de données. La présélection a permis de retenir une trentaine de textes. Des résumés en ont été faits. Chaque résumé a mis en exergue les idées principales des éléments suivants : problématique, objectifs, cadre de référence, méthodologie, résultats et limites de l'étude. Ces informations détaillées sont accessibles dans le rapport de recherche. ${ }^{4}$

La rédaction des résumés de la trentaine de textes présélectionnés a permis de retenir un total de neuf textes correspondant aux trois critères énoncés précédemment. Il est à noter que tout texte dont la structure, explicite ou implicite, s'apparentait à celle d'un écrit scientifique qui présente les résultats d'une expérimentation, a été considéré. Nous n'avons pas tenu compte de critères tels que le lieu de publication (revue avec comité de lecture ou non), le statut de l'auteur (chercheur, professeur, conseiller, consultant, spécialiste, professionnel, enseignant, etc.) ou l'affiliation (université, cégep, école, institut, organisme gouvernemental, etc.). La distribution des textes au regard de leur approche méthodologique correspond à la suivante :

- recherche participative $: \mathrm{n}=1$

- enquêtes : $\mathrm{n}=3$

- études de cas : $\mathrm{n}=5$

Le Tableau 2 présente les textes retenus et leurs principales caractéristiques.

\footnotetext{
${ }^{4}$ Le rapport de recherche complet non publié est accessible à l'adresse http://affordance.uqac.ca/publications/RapportBlogues2010-Final.pdf
} 
Tableau 2: Textes retenus et leurs principales caractéristiques
Id. PROTIC : une pratique

pédagogique porteuse de réussite.

École secondaire Les Compagnons-

de-Cartier. Commission scolaire des

Découvreurs.

Texte Brunsell, E., \& Cimino, C. (2009).

B Investigating the impact of weekly weblog assignments on the environment of a secondary biology course. In Education - Exploring our connective landscape, 15(1).

Texte Clark, C., \& Dugdale, G. (2009).

C Young people's writing: Attitudes, behaviour and the role of technology. London: National Literacy Trust.

Texte De Craene, M. (2005). Pedagogical

D blogs: A pilot study exploring blogs as a learning tool for middle school student with special needs. Mirandanet E-Journal

Texte Luckin, R., Clark, W., Graber, R.,

E Logan, K., Mee, A., \& Martin, O. (2009). Do Web 2.0 tools really open door to learning ? Practices, perceptions and profiles of 11-16year-old students. Learning, Media and Technology, 34(2), 87-104.

Texte Luehmann, A., \& MacBride, R. $F$ (2008). Classroom blogging in the service of student-centered pedagogy: Two high school teachers' use of blogs. THEN: Journal, 1-23.

Texte MacBride, R., \& Luehmann, A. (2008). Capitalizing on emerging
Texte Bélanger, M. (2008). Les blogues en

A $\quad 3^{e}$ secondaire au programme

\section{Type de recherche}

Enquête

Étude de cas

Enquête

cas

Enquête

Étude de

cas

Étude de

\section{Type de} données

Quantitatives

55 élèves de troisième secondaire

\section{Nb de participants}

Qualitatives 36 élèves de $10^{\mathrm{e}}$ année
Quantitatives 3001 élèves provenant de 24 écoles
Qualitatives
5 élèves âgés de 14 ans d'une classe d'adaptation scolaire
Quantitatives - 2611 élèves provenant et de 27 écoles (volet qualitatives quantitatif)
- Quelque 250 élèves provenant de 22 écoles (volet qualitatif)
Qualitatives 2 enseignants et leurs 6 classes

\footnotetext{
${ }^{5}$ http://www.protic.net/
} 


\begin{tabular}{|c|c|c|c|c|}
\hline G & $\begin{array}{l}\text { technologies: A case study of } \\
\text { classroom blogging. School Science } \\
\text { and Mathematics, } 108(5), 173-183 \text {. }\end{array}$ & cas & & \\
\hline $\begin{array}{c}\text { Texte } \\
\mathrm{H}\end{array}$ & $\begin{array}{l}\text { Sousa, S., \& Soares M. (2009). } \\
\text { Developing writing skills through the } \\
\text { use of blogs. L1 - Educational } \\
\text { Studies in Language and Literature, } \\
9(2), 71-90 .\end{array}$ & $\begin{array}{l}\text { Recherche- } \\
\text { action }\end{array}$ & Qualitatives & 13 enseig \\
\hline Texte I & $\begin{array}{l}\text { West, K. (2008). Weblogs and } \\
\text { literary response: Socially situated } \\
\text { identities and hybrid social languages } \\
\text { in English class blogs. Journal of } \\
\text { Adolescent \& Adult Literacy, 51(7), } \\
\text { 588-598. }\end{array}$ & $\begin{array}{l}\text { Étude de } \\
\text { cas }\end{array}$ & Qualitatives & 1 classe \\
\hline
\end{tabular}

À partir des textes retenus et de leurs résumés, trois types de tableaux synthèses ont été élaborés, soit un présentant les cadres de référence employés, le second illustrant les démarches méthodologiques mises de l'avant et un troisième présentant les principaux résultats se dégageant des études retenues. Ce dernier type se décline lui-même en quatre tableaux, soit un pour chacune des dimensions qui ont émergé de l'analyse des textes : a) les conditions affectives; b) les affordances des contextes d'utilisation; c) le déroulement de l'apprentissage et d) les performances.

Il faut souligner que l'amplitude des mots-clés utilisés pour la recherche de textes en a identifié plusieurs qui ne traitaient pas de l'utilisation des médias sociaux. Par exemple, certains abordaient plutôt l'utilisation de didacticiels. Cela explique que nombre de textes aient été mis de côté.

De plus, parmi les textes qui traitaient de l'utilisation du blogue, il importe de spécifier que plusieurs types de documents ont été exclus, dont les articles non scientifiques, les essais, les documents à caractère pédagogique, les témoignages expérientiels, les textes présentant des descriptions d'outils ou des scénarios d'intégration technologique, ainsi que ceux visant à convaincre de la pertinence d'utiliser ou non cette TIC. En outre, nombre de textes à caractère scientifique ont aussi dû être mis de côté compte tenu que les expérimentations conduites l'ont été en milieu universitaire ou dans un contexte de formation continue des enseignants.

\section{Synthèse des connaissances}

La présente partie synthétise et discute des résultats des textes recensés dans le cadre de cette étude. De l'analyse de contenu, émergent quatre principales dimensions autour desquelles la présentation des résultats s'articule : a) les conditions affectives; b) les affordances des contextes d'utilisation; c) le déroulement de l'apprentissage et d) les performances. D'entrée de jeu, chaque dimension est brièvement expliquée. Par la suite, elle est mise en relation avec les textes recensés et des parallèles sont aussi établis avec d'autres éléments des écrits scientifiques. 


\section{Conditions affectives}

Les conditions affectives jouent un rôle important dans l'apprentissage en général (Archambault \& Chouinard, 2009; Bandura, 1997; Pintrich \& Schunk, 1996; Viau, 1994) et il en est ainsi également dans l'apprentissage du langage écrit (Viau, 1999). À ce propos, différents auteurs (Freebody \& Freiberg, 2001; Snow, Burns, \& Griffith, 1998; Turcotte, 2007) s'entendent pour dire que les conditions affectives font référence au sentiment de compétence, à la motivation ainsi qu'au plaisir. Le sentiment de compétence (Harter, Whitesell-Rumbaugh, \& Kowalski, 1992) renvoie au contrôle exercé sur l'apprentissage et sur la confiance en la capacité d'exécuter ou non une tâche avec succès. Le concept de soi (Bong \& Skaalvik, 2003; L'Écuyer, 1978; Marsh, 1990; Ross, 1973; Thomas, 1980) est une notion qui s'y apparente tout en s'en distinguant du fait qu'il réfère à l'estime qu'une personne a d'elle-même, estime qui est susceptible d'être influencée par ses réussites et ses échecs. Quant à la motivation, elle possède plusieurs sources (Deci, Koestner, \& Ryan, 2002; Deci \& Ryan, 1987; Nolen, 2007; Ryan \& Deci, 2000). La motivation intrinsèque, reconnue comme étant la plus propice à un engagement durable dans une tâche, trouve sa source, notamment, dans les désirs, les champs d'intérêt et les objectifs personnels de l'apprenant. En ce qui a trait au plaisir, il fait référence aux sentiments positifs qui incitent à vouloir poursuivre ou revivre une expérience donnée. Enfin, Chartrand et Prince (2009) soulignent l'influence des contextes (école, discipline, tâche, etc.) sur l'intérêt manifesté par les élèves à l'égard des activités qui leur sont proposées.

Le Tableau 3 regroupe les éléments des textes recensés qui réfèrent à ce type de résultats. 
Tableau 3: Résultats et constats pour la dimension "Conditions affectives "

\begin{tabular}{|c|c|}
\hline Constats et résultats & Texte \\
\hline Aucun constat ou résultat pour cette dimension & $\mathrm{H}$ \\
\hline $\begin{array}{l}\text { - } 96 \% \text { des élèves ont affirmé que l'utilisation du blogue a été une activité scolaire } \\
\text { intéressante. } \\
\text { - Peu importe leur goût pour l'écriture, les élèves ont apprécié l'utilisation du blogue. } \\
\text { - } 86 \% \text { des élèves ont dit préférer une utilisation volontaire du blogue, c'est-à-dire } \\
\text { lorsqu'aucune contrainte ou exigence n'y est associée. } \\
\text { - L'utilisation du blogue a plu autant aux élèves qui avaient une plus faible confiance } \\
\text { en leurs aptitudes scolaires qu'à ceux dont la confiance était plus élevée. }\end{array}$ & A \\
\hline $\begin{array}{l}\text { - Les jeunes qui écrivent sur un blogue ont dit que cela augmente leur goût pour } \\
\text { l'écriture. } \\
\text { - Comparativement aux jeunes qui n'utilisent pas de blogue, ceux qui en utilisent un } \\
\text { ont dit préférer écrire. Ils ont aussi une attitude plus positive à l'égard de l'écriture. }\end{array}$ & $\mathrm{C}$ \\
\hline - Tous les élèves ont dit avoir apprécié l'utilisation du blogue. & $\mathrm{D}$ \\
\hline $\begin{array}{l}\text { - Au début de l'expérimentation, } 44 \% \text { des élèves avaient une perception négative de } \\
\text { l'utilisation du blogue, principalement parce qu'ils avaient l'impression qu'elle } \\
\text { augmenterait leur charge de travail. } \\
\text { - } 94 \% \text { des élèves ont dit qu'ils étaient plus enclins à partager leurs idées sur le blogue } \\
\text { qu'en classe. } \\
\text { - Les élèves se sont sentis plus confiants pour fournir des explications en lien avec le } \\
\text { cours. } \\
72 \% \text { des élèves ont dit que l'utilisation du blogue les avait rendus plus confiants dans } \\
\text { leur participation en classe. } \\
\text { - } 94 \% \text { des élèves se sont sentis supportés par leurs pairs dans leur utilisation du } \\
\text { blogue. } \\
97 \% \text { des élèves considèrent que leurs points de vue et leurs opinions ont été valorisés } \\
\text { au sein de la classe. }\end{array}$ & B \\
\hline Aucun constat ou résultat pour cette dimension & F \\
\hline - La dimension publique du blogue a alimenté l'intérêt des élèves. & G \\
\hline $\begin{array}{l}\text { - Les élèves qui ont déjà utilisé un blogue en classe ont dit être motivés par l'utilisation } \\
\text { de cet outil. } \\
\text { - } 66 \% \text { des élèves se sont dits neutres ou non intéressés par une éventuelle utilisation } \\
\text { du blogue en classe. }\end{array}$ & $\mathrm{E}$ \\
\hline $\begin{array}{l}\text { - L'enseignant a eu davantage l'impression de connaitre ses élèves. } \\
\text { - Un niveau d'engagement important a été constaté chez les élèves. }\end{array}$ & I \\
\hline
\end{tabular}

Globalement, on remarque un consensus marqué par rapport à la dimension des conditions affectives. En effet, les résultats des études recensées mettent en lumière de façon quasi unanime la motivation et l'appréciation des élèves à utiliser un blogue en contexte scolaire. La partie traitant des affordances des contextes d'utilisation permettra de mieux comprendre les caractéristiques pouvant contribuer à une telle motivation mais, de prime abord, la dimension non contraignante de l'utilisation de l'outil appert comme un facteur important. Le bémol 
apporté par l'étude de Brunsell et Cimino (2009) (texte B) à l'effet qu'une proportion considérable d'élèves a accueilli timidement cet usage suggère l'importance d'en expliquer aux élèves, d'entrée de jeu, la pertinence à des fins d'apprentissage.

Par souci de prudence, le constat encourageant qui se dégage à propos de la motivation mérite toutefois d'être mis en perspective au regard de la durée des expérimentations menées. À l'exception de celle de Bélanger (2008) (texte A), leur durée a été plutôt courte, soit quelques semaines, voire quelques cours. Un effet de la nouveauté, c'est-à-dire l'effet qui dénote un changement provisoire de réponse des participants lorsqu'ils font face pour la première fois à un contexte donné, est donc possible. En outre, bien qu'on dispose de peu d'informations à propos de la façon dont les contextes d'expérimentation ont été présentés aux élèves, la possibilité d'un effet Hawtorne n'est pas non plus à écarter. Celui-ci réfère à tout changement du niveau de réponse des participants attribuable au simple fait de participer à une expérimentation.

Un autre constat qui émane de résultats convergents a trait au goût pour l'écriture auquel l'utilisation du blogue a contribué. Les élèves semblent y avoir trouvé une source de motivation intrinsèque. Cet intérêt marqué pour l'écriture est relaté de différentes façons. L'enquête de Clark et Dugdale (2009) (texte C), menée auprès de quelque 3000 élèves, révèle que le blogue contribue à l'augmentation de cet intérêt. Bélanger (2008) (texte A) a quant à lui remarqué que, peu importe l'intérêt des élèves pour l'écriture en général (qu'il soit faible ou élevé), ceux-ci ont apprécié leur expérience de rédaction à l'aide d'un blogue. En dépit du petit échantillon de l'étude, il est néanmoins intéressant de constater que l'utilisation du blogue ne semble pas avoir contribué à alimenter un certain effet Matthew, c'est-à-dire augmenter l'écart entre les élèves qui ont un niveau d'intérêt différent pour l'écriture.

Un troisième constat qui émerge de la convergence des résultats a trait à la confiance et au sentiment de compétence que les élèves ont éprouvés dans le cadre du contexte d'utilisation du blogue. Les résultats de l'étude de Brunsell et Cimino (2009) (texte B) sont éloquents à cet effet alors qu'ils mettent en relief que la presque totalité des élèves se sont sentis plus confiants de partager des idées et des explications sur le blogue qu'en classe. Un tel constat rejoint nombre d'autres écrits que ceux recensés et qui expliquent que, de façon générale, la mise à contribution de technologies d'écriture asynchrone favorise une répartition plus équitable du temps de parole entre les apprenants que les situations de communication en face à face. Cela est d'autant plus important que la verbalisation d'idées et d'explications est cruciale pour la construction personnelle des connaissances (Levina, 1981). Malheureusement, l'étude de Brunsell et Cimino (2009) (texte B) rend peu compte de la nature et de la teneur des idées exprimées et construites dans le contexte de leur étude.

Néanmoins, les résultats obtenus à propos du sentiment de confiance et de compétence des élèves sont encourageants puisqu'ils laissent entrevoir que l'utilisation du blogue peut contribuer à faire en sorte que les élèves abordent de façon positive l'écriture, une activité parfois perçue comme étant aversive et fastidieuse (Atwell, 2002).

D'autres résultats, bien qu'ils ne soient pas récurrents d'une étude à l'autre, méritent toutefois d'être soulignés. C'est le cas notamment du sentiment de soutien ressenti par les élèves de l'étude de Brunsell et Cimino (2009) (texte B) qui laisse entrevoir que l'utilisation du blogue pourrait contribuer à encourager, voire faciliter l'expression des difficultés rencontrées par les élèves. Le texte n'aborde pas explicitement cette question, mais le constat suggère une utilisation 
de type diagnostic permettant à l'enseignant d'identifier des éléments de compréhension névralgiques pour ensuite y intervenir de façon spécifique, voire procéder à une certaine remédiation. Similairement, la perception de l'enseignant de l'étude de West (2008) (texte I) quant à une connaissance accrue de ses élèves ouvre une voie intéressante en termes de différenciation pédagogique, bien que, ici aussi, le texte n'aborde pas cette question.

En guise de conclusion à la dimension des conditions affectives, nous retenons que l'utilisation du blogue a été appréciée et jugée motivante par plusieurs groupes d'élèves. Ce constat n'est cependant pas nouveau et il rejoint nombre de travaux (Becta, 2000, 2001a, 2001b; Cox, 1997; Denning, 1997; Denning \& Fisher, 2001; Passey, 2001; Scrimshaw, 1997; Sharples, Graber, Harrison, \& Logan, 2009; Watson, 1993) qui ont montré que d'autres TIC sont un objet de motivation pour les élèves et que ceux-ci apprécient généralement les utiliser en contexte scolaire.

\section{Affordances des contextes d'utilisation}

Le concept d'affordance (Gaver, 1991; Norman, 1983) réfère aux possibilités qui sont présentes dans un environnement et qui incitent des individus à y interagir d'une façon donnée. Par exemple, des études s'intéressent aux propriétés spécifiques de l'écriture électronique, qui est de plus en plus présente à l'ère des nouveaux médias (Kress, 2003; Lee, 2007). Dans le cadre des études recensées, l'environnement réfère aux différents contextes d'intégration du blogue qui ont été documentés. Ces contextes possèdent des attributs, des caractéristiques propres, qu'elles aient été réfléchies et prévues intentionnellement ou non. En d'autres mots, elles suggèrent aux enseignants et aux élèves qui travaillent et apprennent dans un environnement donné des façons d'agir, de l'utiliser. Ces caractéristiques peuvent varier d'un environnement à l'autre et, par conséquent, induire des façons différentes d'y interagir. Bref, une affordance, telle que sa racine anglophone to afford le laisse entendre (Gibson, 1979), c'est ce qui est « offert », rendu possible par un contexte donné, qu'il soit technologique ou non.

La présente dimension rend compte des résultats qui mettent en relief ce qui est rendu possible par les contextes d'utilisation du blogue en classe. Le Tableau 4 regroupe les éléments des textes recensés qui concernent ce type de résultats. 
Tableau 4: Résultats et constats pour la dimension "Affordances des contextes d'utilisation "

\begin{tabular}{|c|c|}
\hline Constats et résultats & Texte \\
\hline $\begin{array}{l}\text { - La dimension publique du blogue a amené les élèves à tempérer la façon d'exprimer } \\
\text { des points de vue négatifs. } \\
\text { - L'utilisation du blogue a permis d'observer, en contexte, le déploiement d'habilités } \\
\text { sociales, cognitives et linguistiques des élèves. }\end{array}$ & $\mathrm{H}$ \\
\hline $\begin{array}{l}\text { - } 96 \% \text { des élèves ont trouvé que le blogue était un outil facile d'utilisation. } \\
\text { - L'utilisation du blogue a procuré un contexte d'écriture authentique. } \\
\text { - L'utilisation du blogue favorise le développement d'une communauté } \\
\text { d'apprentissage. }\end{array}$ & A \\
\hline $\begin{array}{l}\text { - Les jeunes qui écrivent sur un blogue ont dit que cela les soutient dans le } \\
\text { développement de la compétence à écrire. }\end{array}$ & $\mathrm{C}$ \\
\hline $\begin{array}{l}\text { - L'utilisation du blogue procure un contrôle sur la démarche d'écriture aux élèves. } \\
\text { - L'utilisation du blogue encourage les élèves à devenir des producteurs de contenus. } \\
\text { - L'utilisation du blogue modifie la relation élèves-enseignant en faisant en sorte que } \\
\text { ce dernier est devenu davantage un facilitateur. } \\
\text { - L'utilisation du blogue procure un contexte d'écriture authentique. } \\
\text { - L'utilisation du blogue met à la disposition des élèves un contexte favorisant la } \\
\text { réflexion sur leur démarche d'écriture. }\end{array}$ & $\mathrm{D}$ \\
\hline Aucun constat ou résultat pour cette dimension & $\mathrm{B}$ \\
\hline $\begin{array}{l}\text { - Le blogue constitue un outil flexible pouvant être utilisé aux fins d'une diversité } \\
\text { d'intentions pédagogiques. } \\
\text { - L'utilisation du blogue favorise une compréhension en profondeur. } \\
\text { - L'utilisation du blogue favorise le développement d'un sens de la communauté. } \\
\text { - L'utilisation du blogue permet d'ouvrir la classe sur le monde. } \\
\text { - L'utilisation du blogue valorise l'expression des élèves. } \\
\text { - L'utilisation du blogue fournit à l'enseignant un accès à la compréhension des élèves. } \\
\text { - L'utilisation du blogue procure un contrôle de l'apprentissage aux élèves. }\end{array}$ & $\mathrm{F}$ \\
\hline $\begin{array}{l}\text { - L'utilisation du blogue permet d'ouvrir la classe sur le monde. } \\
\text { - L'utilisation du blogue a permis d'appuyer le développement des connaissances } \\
\text { mathématiques des élèves sur leurs habiletés technologiques. }\end{array}$ & G \\
\hline $\begin{array}{l}\text { - Une utilisation pédagogique du blogue passe par l'importance de l'accompagnement } \\
\text { fourni aux élèves par l'enseignant. } \\
\text { Dans les situations où les élèves étaient accompagnés par un enseignant pendant } \\
\text { l'utilisation du blogue, les élèves ont perçu davantage le potentiel de discussion et } \\
\text { d'échange d'idées avec les pairs que dans les situations où les élèves ne bénéficiaient } \\
\text { pas d'accompagnement. }\end{array}$ & $\mathrm{E}$ \\
\hline $\begin{array}{l}\text { - L'utilisation du blogue modifie la relation élèves-enseignant en faisant en sorte que } \\
\text { ce dernier n'est plus qu'un transmetteur d'informations; il participe à l'activité } \\
\text { d'apprentissage avec les élèves. }\end{array}$ & I \\
\hline
\end{tabular}


Tout comme ce fut le cas pour la dimension des conditions affectives, les résultats de plusieurs études convergent ici aussi. Un premier constat qui émane de la plupart des textes recensés a trait à la facilité d'utilisation technologique de l'outil. Il s'agit d'un outil souple et rapide pour diffuser du contenu sur Internet. Les études de Luehmann et MacBride (2008) (texte F) et de De Craene (2005) (texte D) mettent d'ailleurs bien en valeur le fait que l'utilisation du blogue valorise l'expression des élèves et les amène à adopter davantage un rôle de producteurs de contenus. Dans l'ensemble, les affordances et les caractéristiques pédagogiques de l'outil sont cependant peu discutées.

Un second constat qui ressort concerne le contexte d'écriture authentique offert par l'utilisation du blogue. Cet aspect est particulièrement présent dans les écrits de Bélanger (2008) (texte A) et de De Craene (2005) (texte D). Cette affordance procure un auditoire réel aux élèves, ce qui les amènerait à trouver un sens accru à la tâche d'écriture, mais aussi, selon l'étude de Sousa et Soares (2009) (texte H), à tempérer certains points de vue délicats partagés. Une compréhension de la pertinence d'une activité est reconnue comme ayant des incidences positives sur l'engagement des élèves dans l'apprentissage (Hidi, 2001; Schiefele, 2001). En outre, des auteurs (Luehmann \& MacBride, 2008 (texte F); MacBride \& Luehmann, 2008 (texte G)) mentionnent que l'utilisation du blogue procure à la classe une véritable ouverture sur le monde. Certes, le caractère public du blogue, qui est presque omniprésent dans l'ensemble des études recensées, fait en sorte que les écrits qui y sont partagés sont accessibles à quiconque sur Internet. Or, des études apportent certains bémols en relatant que les commentaires provenant de l'extérieur de la classe ne sont pas fréquents, voire que leur contenu manque de consistance (MacBride \& Luehmann, 2008 (texte G)). Bien que cela ne soit pas documenté par les études recensées, un tel constat est à même de questionner la portée réelle du contexte d'écriture authentique dans une optique d'utilisation du blogue à plus long terme. Cet aspect demeure à documenter.

Un troisième résultat récurrent à travers les textes recensés a trait au potentiel de l'utilisation du blogue pour le développement d'un sens de la communauté au sein de la classe. Les textes de Luehmann et MacBride (2008) (texte F) et de Bélanger (2008) (texte A) sont ceux qui abordent le plus spécifiquement cette affordance. Le contexte d'apprentissage mis en place fournirait un ancrage commun qui contribuerait à alimenter une certaine solidarité entre les élèves. Le texte de Luehmann et MacBride (2008) (texte F) souligne que cela favoriserait aussi une compréhension en profondeur. Or, peu de données empiriques sont fournies dans le texte pour soutenir cette assertion.

En quatrième lieu, le contrôle sur la démarche d'apprentissage offert aux élèves par les contextes d'utilisation du blogue qui ont été recensés constitue une autre caractéristique récurrente qui peut être considérée à titre d'affordance. Ce contrôle est reconnu comme un facteur d'engagement dans l'apprentissage (Archambault \& Chouinard, 2009; Connell, 1990; Powell \& Nelson, 1997; Ross, 1988). Ce que les études de De Craene (2005) (texte D) et Luehmann et MacBride (2008) (texte F) mettent en lumière, notamment, c'est l'aspect libertaire que la participation au blogue procure aux élèves. Ce constat rejoint en quelque sorte une orientation récente de la recherche sur l'écriture, soit celle de l'écriture libre (freewriting) (Hayes, 2006). En outre, les élèves ont le sentiment qu'ils sont responsables de ce à quoi ils prennent part, qu'ils y sont des acteurs actifs et engagés. Cela dit, l'étude de Luckin et al. (2009) (texte E) met bien en garde contre une liberté absolue accordée aux élèves et une mise à contribution qui ne serait pas guidée par une intention pédagogique. Ils arguent l'importance de l'accompagnement de l'enseignant, autant en ce qui a 
trait à la planification de l'utilisation du blogue à des fins pédagogiques qu'à celui offert pendant son utilisation.

Le rôle de l'enseignant constitue un cinquième pan de résultats convergents qui émane des contextes d'utilisation du blogue recensés. La pluralité de ces contextes suggère que l'outil offre suffisamment de souplesse pour répondre à une diversité d'intentions pédagogiques, bien que peu d'entre elles aient été explicitement mentionnées dans les textes. En outre, les textes de De Craene (2005) (texte D) et de West (2008) (texte I) évoquent une modification des types de relations en classe en mentionnant que l'enseignant est davantage porté à adopter une posture de facilitateur et d'accompagnateur, et qu'un partage d'informations s'effectue entre les élèves. De tels résultats convergent dans une certaine mesure avec les interventions contributoires aux échanges entre pairs recensées par Cooper \& Brna (2002). En outre, il serait intéressant d'en connaitre davantage à propos du contexte de développement professionnel des enseignants ayant participé à ces études pour pouvoir mettre en relation ces dernières avec celle de Becker et Riel (1999) qui a identifié que les enseignants qui prennent part à des activités de développement professionnel sont ceux qui sont les plus susceptibles d'adopter des pratiques d'intégration des TIC constructivistes.

Bien que la nature des interventions effectuées par les enseignants sur le blogue soit peu documentée de façon détaillée, les textes de Luehmann et MacBride (2008) (texte F) et de Sousa et Soares (2009) (texte H) traitent néanmoins sommairement du changement de rôle de l'enseignant. Le premier mentionne que l'utilisation du blogue invite l'enseignant à saisir la compréhension que les élèves se forgent et dont ils rendent compte par le biais de l'écrit. Le second texte soutient quant à lui que le contexte d'utilisation du blogue convie à une position d'observateur du déploiement de la capacité des élèves en contexte réel. En ce sens, on peut dire qu'il y a une certaine décentration du rôle de l'enseignant vers l'élève.

En conclusion à cette dimension, nous retenons que les contextes d'utilisation du blogue peuvent offrir un apprentissage authentique aux élèves, ainsi qu'un contrôle sur cet apprentissage, qu'ils sont en support au développement d'une relation de groupe au sein de la classe et qu'ils invitent à une modification de la relation élèves-enseignant dans le rapport à la connaissance.

\section{Déroulement de l'apprentissage et performances}

La dimension du déroulement de l'apprentissage réfère à la façon dont le blogue a été mis à contribution, à l'organisation du contexte dans lequel il a été intégré et à ce que les acteurs impliqués y ont fait en cours d'utilisation.

Cette dimension a aussi été observée du point de vue plus spécifique du développement de l'écriture. Cette dernière est une tâche complexe qui requiert du scripteur la mobilisation de différents types de savoirs, tels que des connaissances générales, des connaissances de la langue et des connaissances liées à la tâche à réaliser (Barré-De Miniac, 2000). Or, comme le précise Préfontaine (1998), à une certaine époque, peu d'importance était accordée aux processus et aux stratégies d'écriture. Par conséquent, il n'était pas enseigné aux élèves comment trouver des idées originales et pertinentes, déterminer une intention d'écriture et un destinataire, rédiger un brouillon et réviser un texte. L'écriture en contexte scolaire signifiait essentiellement produire un texte selon un thème précisé par l'enseignant et dont il était le seul destinataire. Une fois 
corrigées et annotées, les productions étaient remises aux élèves et il n'y avait aucun retour sur le travail effectué (Rijlaarsdam et al., 2008).

Or, selon la tâche à réaliser, le scripteur devrait plutôt déployer des stratégies liées aux différents processus d'écriture : planifier, rédiger et réviser. Puisque cette démarche n'est pas linéaire, il est donc possible de revenir sur ce qui a été fait et d'anticiper le travail à réaliser. Ainsi, selon l'intention d'écriture et le destinataire, les connaissances sont activées et un plan d'écriture est établi. Lors de la rédaction, le scripteur s'assure de la pertinence et de la clarté des idées. L'amélioration des idées et la vérification des conventions linguistiques constituent les éléments pris en compte au moment de la révision. Cette démarche sera complétée par la diffusion du produit final sur un support approprié (Hayes, 1995; Hayes \& Flower, 1980; Nadon, 2007; Simard, 1995). Enfin, la compétence à écrire se développe par la réalisation d'activités se rapprochant de situations de la vie quotidienne (Gagnon \& Ziarko, 2005).

La dimension des performances fait quant à elle référence aux résultats des écrits recensés qui traitent du produit, de la résultante, de l'extrant et des retombées des contextes d'utilisation du blogue.

Le Tableau 5 regroupe les résultats relatifs à la dimension du déroulement de l'apprentissage, issus des textes retenus dans le cadre de la recension. 
Tableau 5: Résultats et constats pour la dimension " Déroulement de l'apprentissage "

\begin{tabular}{|c|c|}
\hline Constats et résultats & Texte \\
\hline $\begin{array}{l}\text { - L'utilisation du blogue a amené les élèves à mettre en œuvre une démarche de } \\
\text { production, d'évaluation et de réécriture de textes. }\end{array}$ & $\mathrm{H}$ \\
\hline $\begin{array}{l}\text { - } 96 \% \text { des élèves ont dit s'appliquer davantage à l'écrit lorsqu'ils utilisent le blogue. } \\
\text { - } 100 \% \text { des élèves ont jugé que le blogue est un outil pertinent pour l'apprentissage du } \\
\text { français. } \\
\text { - Plus de } 66 \% \text { des élèves ont jugé que le blogue est un outil pertinent pour } \\
\text { l'apprentissage de l'anglais et de l'histoire. }\end{array}$ & A \\
\hline Aucun constat ou résultat pour cette dimension & $\mathrm{C}$ \\
\hline $\begin{array}{l}\text { - Un élève a utilisé un dictionnaire pendant qu'elle écrivait sur le blogue. } \\
\text { - } 94 \% \text { des élèves ont dit que l'utilisation du blogue les a amenés à effectuer des } \\
\text { recherches personnelles supplémentaires en lien avec le sujet du cours. } \\
\text { - } 86 \% \text { des élèves ont dit qu'ils poursuivront des recherches personnelles } \\
\text { supplémentaires dans d'autres cours. }\end{array}$ & $\mathrm{D}$ \\
\hline $\begin{array}{l}\text { - Plus de } 80 \% \text { des élèves ont estimé que la participation au blogue les a aidés à se } \\
\text { préparer aux examens en classe. }\end{array}$ & $\mathrm{B}$ \\
\hline $\begin{array}{l}\text { - } 6 \text { types d'utilisations du blogue ont été identifiés : } \\
\circ \text { Partage de ressources } \\
\circ \text { Réponse aux questions de l'enseignant } \\
\circ \text { Prise de notes } \\
\circ \text { Partage de défis d'apprentissage } \\
\circ \text { Réflexion sur ce qui a été appris } \\
\circ \text { Conversation en ligne } \\
\end{array}$ & $\mathrm{F}$ \\
\hline $\begin{array}{l}\text { - Peu d'indices à l'effet que les élèves ont amélioré les textes écrits avec le blogue ont } \\
\text { été observés. } \\
\text { - Le blogue a servi d'outil de révision en prévision des examens. } \\
\text { - La lecture du blogue collectif est devenue une routine de classe. }\end{array}$ & $\mathrm{G}$ \\
\hline $\begin{array}{l}\text { - } 3 \text { types d'activités ont été recensés } \\
\circ \text { Recherche d'informations (peu de preuves que cela est effectué en exerçant } \\
\text { un jugement critique). } \\
\circ \quad \text { Collaboration (peu d'exemples relatent de la coconstruction de } \\
\text { connaissances). } \\
\quad \text { Producteurs de contenus (partage d'expériences, d'opinions, etc.). } \\
\text { - Les élèves dépendent essentiellement de leurs enseignants en ce qui a trait à la } \\
\text { sélection de l'information. }\end{array}$ & E \\
\hline $\begin{array}{l}\text { - L'enseignant avait l'impression que les élèves comprenaient davantage le processus } \\
\text { de réflexion et de lecture des uns et des autres. }\end{array}$ & I \\
\hline
\end{tabular}

Nous dégageons ici deux principaux constats convergents à travers les écrits. Le premier s'inscrit dans la foulée de la diversité d'intentions pédagogiques pouvant être soutenues par l'utilisation du blogue, tel que discutées à la dimension précédente. Au regard du déroulement, une diversité d'usages du blogue a été constatée, dont le partage de ressources, la prise de notes, la réflexion sur les apprentissages effectués, la conversation en ligne, la préparation aux examens, le compte 
rendu de recherches d'informations. Cela suggère une souplesse et une flexibilité de l'outil à des fins pédagogiques. Néanmoins, les études recensées ont, dans l'ensemble, peu abordé la nature, la dimension qualitative des contenus écrits à l'aide du blogue. De plus, on en connaît peu à propos de la façon dont l'utilisation du blogue a été arrimée aux activités en face à face de la classe. Seules les études de Brunsell et Cimino (2009) (texte B) et de MacBride et Luehmann (2008) (texte G) offrent quelques informations à cet effet, en précisant, respectivement, que le blogue a servi à compléter un devoir hebdomadaire qui faisait suite aux notions abordées en classe, et qu'il est devenu une routine de classe, tout en étant utilisé à des fins de préparation à l'examen, grâce aux traces qu'il a permis de conserver. Quant au contexte étudié par Bélanger (2008) (texte A), on déduit qu'il y a eu un arrimage serré entre le face à face et l'utilisation du blogue en raison de la forte propension technologique de PROTIC (programme d'intégration des TIC où chaque élève dispose de son propre ordinateur portable) mais, ici aussi, de plus amples informations auraient permis de mieux rendre compte de l'orchestration du contexte d'utilisation du blogue.

Le second élément convergent concerne la pertinence pédagogique reconnue. La lecture d'une majorité de textes recensés suggère que les élèves, tout comme les enseignants, perçoivent dans le blogue un potentiel pour l'apprentissage. Il est intéressant de constater que ce potentiel ne concerne pas que l'apprentissage des langues. Les travaux de Bélanger (2008) (texte A) sont parmi les plus évocateurs à cet effet. L'auteur a relevé, en plus du français et de l'anglais, la pertinence de l'utilisation du blogue en histoire. Les travaux de Luehmann et MacBride (2008) (texte F) et de MacBride et Luehmann (2008) (texte G) relatent des constats semblables en ce qui a trait à l'intégration du blogue dans des classes de mathématique et de biologie.

Par ailleurs, un certain nombre d'éléments divergents ressortent des textes recensés. Le premier a trait au potentiel de révision offert par l'utilisation du blogue, une étape importante de la démarche d'écriture (Hayes, 1995; Hayes \& Flower, 1980). L'étude de Sousa et Soares (2009) (texte $\mathrm{H}$ ) affirme que le contexte d'utilisation du blogue a amené les élèves à s'inscrire dans un processus de réécriture de textes. Or, peu d'informations permettant de comprendre la façon dont cela s'est orchestré sont fournies dans le texte. En contrepartie, le texte de MacBride et Luehmann (2008) (texte G) relate que peu d'indices tangibles leur permettent d'affirmer que les élèves ont amélioré leurs écrits. Pour mieux comprendre les tenants et aboutissants de ces constats divergents, davantage de précisions à propos des contextes d'utilisation mis en place et des affordances des outils utilisés seraient, ici aussi, requises.

En outre, un autre constat divergent à travers les écrits recensés concerne les tâches de recherche et de traitement de l'information prévues dans les contextes d'utilisation des blogues. De Craene (2005) (texte D) constate qu'une proportion importante d'élèves dit être en mesure de poursuivre des recherches personnelles dans le cadre de cours ultérieurs alors que l'étude de Luckin et al. (2009) (texte E) en vient à la conclusion que les élèves dépendaient essentiellement de l'enseignant à cet égard. Il importe cependant de mettre en perspective cette divergence en considérant les approches méthodologiques employées dans le cadre des études concernées par ces résultats. Le texte de De Craene (2005) (texte D) présente une étude de cas. Le contexte étudié est par conséquent beaucoup plus ciblé que l'enquête (Luckin et al., 2009 (texte E)) effectuée auprès de quelque 2600 élèves qui, dans leurs réponses, ont possiblement considéré une vaste gamme de contextes d'apprentissage auxquels ils ont pris part. 
Par ailleurs, des résultats singuliers méritent néanmoins d'être soulignés. Bélanger (2008) (texte A) a constaté que son contexte d'utilisation du blogue amenait la presque totalité des élèves à s'appliquer davantage dans le travail réalisé. Une piste d'explication réside dans sa dimension authentique et dans l'auditoire réel qu'il procure aux élèves, telle que relatée dans la partie des résultats traitant des affordances des contextes d'utilisation. En outre, l'étude de West (2008) (texte I) suggère que l'utilisation du blogue a contribué à une meilleure compréhension du processus d'apprentissage par les élèves. Bien que peu d'éléments explicatifs accompagnent cette conclusion, nous y voyons un potentiel métacognitif intéressant à explorer.

En conclusion à la dimension du déroulement de l'apprentissage, il faut rappeler la diversité des utilisations possibles pouvant être faites du blogue ainsi que sa pertinence pédagogique relatée, autant par les élèves que par les enseignants.

Le Tableau 6 regroupe les résultats relatifs à la dimension des performances, issus des textes retenus dans le cadre de la recension.

Tableau 6: Résultats et constats pour la dimension " Performances"

\begin{tabular}{|c|c|}
\hline Constats et résultats & Texte \\
\hline $\begin{array}{l}\text { - Style d'écriture essentiellement informel, caractérisé par une ponctuation expressive, } \\
\text { l'utilisation d'un langage familier, de l'argot et d'onomatopées. } \\
\text { - Dans les commentaires émis aux billets des autres, les élèves n'ont pas hésité à faire } \\
\text { part de points de vue différents. }\end{array}$ & $\mathrm{H}$ \\
\hline Aucun constat ou résultat pour cette dimension & A \\
\hline - Les jeunes qui possèdent un blogue ont dit écrire davantage que ceux qui n'en ont pas. & $\mathrm{C}$ \\
\hline $\begin{array}{l}\text { - Les élèves ont écrit à propos de ce qu'est la confiance en soi, de ce qui contribue à } \\
\text { l'augmenter et à la diminuer, et des conséquences de sa diminution. } \\
\text { - Présence marquée de langage informel spécifique à la communication électronique } \\
\text { (abréviations, acronymes, émoticônes, etc.). } \\
\text { - Les commentaires aux billets étaient surtout de l'ordre de la rétroaction positive. } \\
\text { - Des élèves ont noté la présence d'erreurs linguistiques dans les billets écrits par leurs } \\
\text { camarades. }\end{array}$ & $\mathrm{D}$ \\
\hline $\begin{array}{l}\text { - Les élèves ont conservé un haut niveau de participation tout au long de la période } \\
\text { d'utilisation du blogue. } \\
\text { - La participation aux échanges en classe a augmenté tout au long de la période } \\
\text { d'utilisation du blogue (de } 17 \% \text { à } 72 \% \text { en huit semaines). }\end{array}$ & B \\
\hline Aucun constat ou résultat pour cette dimension & $\mathrm{F}$ \\
\hline $\begin{array}{l}\text { - Les commentaires émis par des personnes extérieures de la classe étaient de l'ordre de } \\
\text { l'appréciation et de la mise en valeur des billets écrits par les élèves. } \\
\text { - La présence d'explications personnelles de concepts mathématiques a été observée. } \\
\text { - La mise en commun de ressources en mathématiques a été observée. }\end{array}$ & G \\
\hline - Les élèves ont dit surtout consulter les blogues (lecture). & $\mathrm{E}$ \\
\hline $\begin{array}{l}\text { - Les idées individuelles des élèves ont été mises en commun pour en générer de } \\
\text { nouvelles. } \\
\text { - Le non respect des conventions linguistiques de base a été observé. }\end{array}$ & I \\
\hline
\end{tabular}


Trois principaux constats convergent des résultats relatifs aux performances issues des contextes d'utilisation recensés du blogue. D'une part, l'importance du volume d'écriture et de la participation ressort comme un élément consensuel, c'est-à-dire que, globalement, les élèves ont été très loquaces. Ce résultat est intéressant du fait qu'il indique que l'utilisation du blogue peut amener les élèves à écrire une quantité significative de texte, ce qui leur fait prendre part à de nombreuses occasions d'écriture. Cela dit, au-delà de la quantité, tel que mentionné précédemment, on en sait peu à propos de la nature des contenus et des idées - si ce n'est qu'ils sont variés - et de la démarche d'écriture mise en œuvre; des aspects pourtant importants à considérer dans le développement de la compétence à écrire.

D'autre part, certaines études (Sousa \& Soares, 2009 (texte H); De Craene, 2005 (texte D); West, 2008 (texte I)) ont mis en lumière une présence importante de langage informel (abréviations, acronymes, émoticônes, etc.) dans les textes écrits. Cela soulève la question du registre de langue à utiliser dans le cadre de l'utilisation du blogue. L'intervention enseignante appert ici avoir un rôle important à jouer, tel que le rappelle l'étude de Luckin et al. (texte E).

Enfin, si la mise en commun d'idées, de ressources et de points de vue a été fréquemment constatée, la négociation de sens, élément important dans la construction sociale des connaissances, semble toutefois être moins présente. En effet, si l'étude de Sousa et Soares (2009) (texte H) relate que les élèves n'ont pas hésité à faire part d'opinions différentes et qu'ils sont capables de le faire de façon tempérée, celles de De Craene (2005) (texte D) et de MacBride \& Luehmann, (2008) (texte G) ont surtout remarqué que les commentaires soumis étaient davantage de l'ordre de la rétroaction positive, c'est-à-dire pour encourager les élèves dans leur travail et valoriser leurs idées. Bien que de tels commentaires ne soient pas dénudés d'intérêt, force est d'admettre que leur potentiel à des fins de coconstruction demeure limité.

Par ailleurs, quelques constats uniques méritent aussi d'être mis en exergue, soit la présence d'explications de la part des élèves (MacBride \& Luehmann, 2008 (texte G)) et une participation accrue aux échanges en face à face (Brunsell \& Cimino, 2009 (texte B)). Ce dernier constat est particulièrement intéressant du fait qu'il suggère un certain transfert d'un comportement en ligne au sein de la classe physique.

En conclusion à cette dimension, il appert que l'utilisation du blogue ouvre la voie à l'exploration d'aspects plus spécifiques du développement de la compétence à écrire du fait que les élèves se prêtent volontiers à la rédaction de textes par le biais de ce média. Le respect des normes linguistiques semble toutefois un enjeu pédagogique important qui mérite réflexion, tout particulièrement dans le contexte social québécois actuel où la qualité de la langue française écrite fait l'objet de nombreuses discussions. 


\section{Regard sur les aspects conceptuels et méthodologiques des travaux recensés}

Cette partie veut porter un regard panoramique sur les aspects conceptuels et méthodologiques des écrits consultés dans le cadre de cette recension, cela afin d'aiguiller des recherches éventuelles. Il importe de préciser que les réflexions présentées dans cette partie vont au-delà des neuf textes retenus. En effet, à certains égards, elles s'appuient également sur la consultation des textes qui ont été écartés lors de la sélection.

Quelle que soit leur nature - personnelle ou professionnelle - les écrits à propos des blogues sont abondants. Or, West, 2008 (texte I) et MacBride \& Luehmann, 2008 (texte G) constataient le peu de textes scientifiques portant sur les contextes d'apprentissage intégrant le blogue. Quelques années plus tard, la démarche menée dans le cadre de notre recension nous amène à réitérer ce constat, particulièrement en contextes primaire et secondaire. De plus en plus d'études sont toutefois menées en contextes universitaire et de formation continue. En outre, la proportion d'écrits professionnels est impressionnante. Globalement, ils sont de deux types. Le premier cherche à convaincre de la pertinence d'utiliser le blogue, en faisant la plupart du temps appel à des arguments généraux tels que : la société du savoir, le besoin d'innover en éducation, la pertinence de collaborer, de rendre l'élève actif, de s'inscrire dans un nouveau paradigme d'apprentissage faisant la promotion de la construction des connaissances, etc. (Amsen, 2006; Bernstein, 2004; Epelboin \& O’Dell, 2009; Ferriter, 2009; Ramswami, 2008; Tomé, 2009; Ward, 2004). Dans ces cas, peu d'appuis empiriques sont évoqués de façon systématique. On argue souvent le seul besoin de changement à l'école pour justifier la pertinence d'utiliser le blogue.

L'autre type de textes nous semble ouvrir progressivement la voie à un partage et à une explicitation accrus des pratiques enseignantes. En effet, nombre d'écrits professionnels proviennent d'enseignants ou d'autres pédagogues qui rendent compte, de façon descriptive et/ou réflexive, et de façon plus ou moins systématique, indépendamment des auteurs, d'expériences vécues à l'aide des blogues dans leur contexte de travail. Ces écrits expérientiels contribuent à rendre davantage publique, visible, la pratique des pédagogues concernés, un peu à l'instar de ce dont parlent Laferrière, Breuleux et Allaire (2007) à propos du travail en réseau. Ils contribuent aussi potentiellement à alimenter un réseau d'entraide, d'échange et de partage plus ou moins informel.

Au regard des textes à caractère scientifique, soit ceux qui ont été principalement recensés dans le cadre de cette étude, les quelques éléments suivants nous semblent importants à mettre en lumière. D'une part, peu d'articles traitent spécifiquement du développement de la compétence à écrire. En fait, on peut dire que, dans les écrits recensés, le blogue a été documenté en tant qu'outil d'écriture davantage qu'en tant qu'outil permettant le développement de l'écriture. L'étude de Sousa et Soares (2009) (texte H) s'y est intéressée, à tout le moins en partie, mais on ne sait pas de quelle façon exactement.

D'autre part, plusieurs études de cas se réclament d'une perspective théorique constructiviste et socioconstructiviste. Or, elles omettent souvent de présenter un cadre conceptuel permettant d'observer systématiquement les éléments relatifs aux objectifs poursuivis. Il en découle parfois une démarche qui semble avoir été menée essentiellement à tâtons. Cela peut s'expliquer par le fait que, bien que les blogues soient maintenant connus depuis une dizaine d'années, leur pénétration en milieu scolaire demeure relativement récente et peu présente (Roy, 2009). Une 
phase de butinage apparaît avoir été nécessaire aux auteurs recensés pour leur permettre d'identifier des pistes fécondes. Dans le cadre de recherches futures, il nous apparaitrait toutefois important de documenter des aspects plus ciblés pour mettre en lumière de façon plus spécifique et précise le potentiel des contextes d'utilisation du blogue, afin de dépasser l'évocation de constats généraux qui, dans certains cas, ne sont pas liés aux données collectées.

Ensuite, les mesures de performance des contextes d'utilisation du blogue sont fréquemment quantitatives, c'est-à-dire qu'on mesure les extrants de la participation au blogue par le nombre d'entrées, leur longueur, la fréquence d'écriture, etc. Des études offrent toutefois des pistes prometteuses au regard d'indicateurs plus qualitatifs (Luehmann \& MacBride, 2008 (texte F) ; Luckin et al., 2009 (texte E)). Par exemple, on y traite des types de comportements adoptés par différents profils d'utilisateurs et des types d'activités qui se sont déroulées par le biais du blogue. Dans l'ensemble, la dimension qualitative de ce qui s'est écrit sur les blogues scolaires a toutefois été peu documentée à travers les écrits recensés.

En ce qui a trait à la question de l'étayage offert aux élèves, qu'il provienne des affordances de l'outil ou de l'intervention de l'enseignant (voir à cet effet la notion de soft/hard scaffolding de Brush \& Saye, 2002), il est dans l'ensemble peu abordé. Pourtant, le mouvement du ComputerSupported Collaborative Learning, qui s'intéresse à l'utilisation de technologies de collaboration est plutôt consensuel quant à la nécessité d'offrir une forme d'accompagnement en cours de processus (voir notamment les travaux de Koschmann, Myers, Feltovich \& Barrows, 1994 et le numéro spécial de 2004 à propos du concept de scaffolding dans The Journal of the Learning Sciences). On peut en dire tout autant des écrits qui traitent spécifiquement du développement de la compétence à écrire et qui, tel que le fait remarquer Routman (2010), prennent appui, entre autres, sur les écrits de Bruner (1990), Cambourne (1998), Holdaway (2000), Pearson et Taylor (1994) et Vygotsky (1978).

Enfin, l'utilisation du blogue a globalement été étudiée en vase clos des autres activités de la classe. Or, un récent rapport (Means, Toyama, Murphy, Bakia \& Jones, 2009) indique que les contextes d'apprentissage les plus porteurs en termes de réussite scolaire sont ceux qui mettent à profit un environnement d'apprentissage hybride, c'est-à-dire qui combine les interactions en face à face à celles en ligne.

\section{Conclusion}

Cette étude, qui se voulait une façon de donner suite aux travaux du Comité d'experts sur l'apprentissage de l'écriture (MELS, 2008), visait à recenser les travaux à caractère scientifique qui se sont intéressés à l'écriture électronique par le biais du blogue en contextes primaire et secondaire.

Nous jugeons que les résultats qui en émanent font valoir un potentiel pédagogique pour 1'utilisation du blogue dans ce contexte. À cet effet, le caractère authentique que l'utilisation de ce média social confère aux activités d'apprentissage et la motivation que les élèves y trouvent à écrire sont certainement les résultats qui ressortent comme étant les plus marquants. Les contextes d'utilisation du blogue documentés dans cette étude illustrent non seulement que les élèves apprécient écrire ainsi, mais qu'ils peuvent également le faire librement, c'est-à-dire sans 
qu'on ait besoin de les y contraindre. En ce sens, nous y voyons une piste de réponse encourageante à la recommandation suivante du Comité d'experts (MELS, 2008) :

Le Comité recommande que le ministère de l'Éducation, du Loisir et du Sport entreprenne une recherche visant à explorer de quelle façon pourrait être exploité le goût des jeunes pour une écriture spontanée dans le but de développer leur compétence à l'écrit. Une telle recherche devrait viser la conception d'approches pédagogiques innovatrices. (p. 37)

Les résultats encourageants que cette recension a permis de mettre en lumière nous apparaissent cependant avoir besoin d'être étayés de façon plus rigoureuse sur le plan de la recherche pour être en mesure de se prononcer de façon plus affirmative, dans une perspective d'utilisation soutenue et au regard de retombées pour l'apprentissage des élèves et du développement des compétences, en particulier celle de l'écriture. À cet effet, le contenu et la qualité des écrits générés à partir des blogues nous semble être un point d'attention, de même que le faible souci apparent pour la prise en considération des éléments fondamentaux de la démarche d'écriture. En ce sens, pour ce qui est de l'utilisation du blogue, des travaux complémentaires nous apparaissent nécessaires pour répondre positivement à cette autre recommandation du Comité d'experts sur l'écriture (MELS, 2008) :

Le Comité recommande que le ministère de l'Éducation, du Loisir et du Sport encourage des recherches pour mieux adapter l'enseignement de l'écriture et son évaluation à l'environnement technologique des jeunes, pour qui l'informatique est un outil normal de production d'écrits, et pour que soient utilisés de façon efficace les nombreux outils d'aide à la rédaction, à la correction et à la révision des textes. (p. 37)

Dans une perspective de recherche, nous identifions les pistes suivantes dans une optique constructive, pour poursuivre les travaux entamés par des chercheurs qui sont peu nombreux à documenter scientifiquement les pratiques d'écriture électronique à l'aide des médias sociaux au primaire et au secondaire :

- Chercher à documenter des éléments plus spécifiques à partir de cadres de référence étoffés. Des études mènent à des conclusions sans toutefois qu'on sache de quelle façon elles y sont parvenues de façon systématique. Dans certains cas, les conclusions qui sont tirées s'appuient peu sur les données collectées.

- Documenter des contextes d'utilisation du blogue à des fins de développement de la compétence à écrire.

- Mener des expérimentations d'une plus longue durée de sorte à pouvoir poser un regard davantage évolutif et à limiter les impacts de possibles effets Hawtorne et de la nouveauté. Du point de vue de la pratique, si le butinage technologique peut être tentant pour diversifier la nature des activités proposées aux élèves, il en va un peu autrement du point de vue de la recherche, qui requiert un certain approfondissement des pratiques pour mieux en comprendre les tenants et les aboutissants réels.

- Considérant la pénétration récente des médias sociaux dans les écoles, privilégier davantage d'approches de recherche participatives et interventionnistes qui s'ancrent, autant que possible, dans des partenariats université-milieu. De tels partenariats sont reconnus pour leur efficacité dans la mise en place d'innovations en éducation. 
- Tenir compte du contexte d'ensemble des activités de la classe dans l'étude de l'utilisation du blogue.

- Étudier le rôle d'accompagnement de l'enseignant dans les contextes d'utilisation du blogue.

Dans une perspective de design, nous sommes d'avis que le développement d'outils d'analyse et d'observation permettant de documenter l'utilisation du blogue et d'offrir des moyens d'assurer une certaine régulation du travail effectué par les élèves soit une voix à approfondir. De plus, il nous apparait important de chercher à orienter davantage la conception des blogues en se référant à des principes théoriques et pédagogiques reconnus, en plus des préoccupations ergonomiques actuelles (convivialité, facilité d'utilisation, etc.). 


\section{References}

Alexander, B. (2006). Web 2.0: A new wave of innovation for teaching and learning. EDUCAUSE Review. 41(2), 32-44. Récupéré de http://www.educause.edu/apps/er/erm06/erm0621.asp

Allaire, S., Thériault, P., \& Lalancette, E. (2010). L'utilisation du blogue en milieux scolaires primaire et secondaire : des initiatives prometteuses à étayer au plan de la recherche. Recension des écrits (2004-2010). Ministère de l'Éducation, du Loisir et du Sport. Saguenay, QC: Université du Québec à Chicoutimi. Récupéré de http://affordance.uqac.ca/publications/RapportBlogues2010-Final.pdf

Allington, R. L. (2010). Writing difficulties. In A-M. McGill-Franzen \& R.L. Allington, R.L. (Eds.), Handbook or Reading disability research (pp.232-241). New York : Routledge.

Allington, R., \& Cunningham, L. (1998). Classrooms that work: They can all read and write. New York : Longman.

Amsen, E. (2006). Who benefits from science blogging? Hypothesis, 4(2), 10-14.

Archambault, J., \& Chouinard, R. (2009). Vers une gestion éducative de la classe. 3e édition. Boucherville : Gaëtan Morin.

Atwell, N. (1998). In the middle : New understanding about writing, reading and learning. Toronto : Pearson Education Canada.

Atwell, N. (2002). Lessons that change writers. Portsmouth, NH : Heinemann.

Bandura, A. (1997). Self-efficacy: The exercise of control. New York : Freeman.

Barré-De Miniac, C. (2000). Le rapport à l'écriture. Aspects théoriques et didactiques. Villeneuve d'Ascq : Presses Universitaires du Septentrion.

Becker, H. J., \& Riel, M. M. (1999). Teacher professionalism, school work culture and the emergence of constructivist-compatible pedagogies. University of California, Irvine: Center for Research on Information Technology and Organizations.

Becta. (2000). A preliminary report for the DfEE on the relationship between ICT and primary school standards. Coventry, UK: Becta.

Becta. (2001a). Primary schools of the future - achieving today. Coventry, UK: Becta.

Becta. (2001b). The secondary school of the future - A preliminary report to the DfEE by Becta. Coventry, UK: Becta.

Bélanger, M. (2008). Les blogues en $3^{e}$ secondaire au programme PROCTIC : une pratique pédagogique porteuse de réussite. École secondaire Les Compagnons-de-Cartier. Commission scolaire des Découvreurs. Récupéré de http://www.proticdocs.net/martinbelanger/public/rapport_blogage_v2.pdf 
Bernstein, M. (2004). Do weblogs improve writing? Récupéré de http://markbernstein.org/Jan0401.html\#note_35302

Bong, M., \& Skaalvik, E. (2003). Academic self-concept and self-efficacy: How different are they really? Educational Psychology Review, 15(1), 1-40.

Boyd, D. (2006). A blogger's blog: Exploring the definition of a medium, Reconstruction 6.4. Récupéré de http://reconstruction.eserver.org/064/boyd/shtml

Bruner, J. (1990). Acts of meaning. Cambridge, MA : Harvard University Press.

Brunsell, E., \& Cimino, C. (2009). Investigating the impact of weekly weblog assignments on the environment of a secondary biology course. In Education - Exploring our connective landscape, 15(1).

Brush, T., \& Saye, J. (2002). A Summary of research exploring hard and soft scaffolding for teachers and students using a multimedia supported learning environment. The Journal of Interactive Online Learning, 1(2).

Cambourne, B. (1998). The whole story: Natural learning and the acquisition of literacy learning in the classroom. Auckland, NZ: Ashton Scholastic.

Chartrand, S., \& Prince, M. (2009). La dimension affective du rapport à l'écrit d'élèves québécois. Revue canadienne de l'éducation, 32(2), 317-343.

Clark, C., \& Dugdale, G. (2009). Young people's writing : Attitudes, behaviour and the role of technology. London: National Literacy Trust.

Cochran-Smith, M., Paris, C. L., \& Kahn, J.L. (1991). Learning to write differently: Beginning writers and word processing. Norwood, NJ: Ablex Publishing.

Coker, D., \& Lewis, W. E. (2008). Beyond writing next: A discussion of writing research and instructional uncertainty. Harvard Educational Review, 78(1), 231.

Connell, J. (1990). Context, self, and action: A motivational analysis of self-esteem process across life-span. In D. Ciccheti (Ed.), The self in transition: Infancy to childhood. Chicago: University of Chicago Press.

Cooper, B., \& Brna, P. (2002). Supporting high quality interaction and motivation in the classroom using ICT: the social and emotional learning and engagement in the NIMIS project. Education, Communication and Information, 2(2-3), 113-138.

Cox, M. (1997). The effects of information technology on students' motivation. London: Kings College London and Coventry.

Daiute, C. (1983). The computer as stylus and audience. College Composition and Communication, 34, 34-145.

Daiute, C. (1985). Writing and computers. Reading, MA: Addison-Wesley. 
Deci, E. L., Koestner, R., \& Ryan, R. M. (2001). Extrinsic rewards and intrinsic motivation in education: Reconsidered once again. Review of Educational Research, 71(1), 1-27.

Deci, E., \& Ryan, R. (1987). The support of autonomy and the control of behavior. Journal of Personality and Social Psychology, 53, 1024-1037.

De Craene, M. (2005). Pedagogical blogs: A pilot study exploring blogs as a learning tool for middle school student with special needs. Mirandanet E-Journal.

Denning, T. (1997). IT and pupil motivation: A collaborative study of staff and pupil attitudes and experiences. Stoke-on-Trent, UK: Keele University and Coventry.

Denning, T., \& Fisher, T. (2001). On-line courses for key stage 3 in Japanese, Latin and mathematics. Coventry, UK: Becta.

Dickinson, D. K. (1986). Cooperation, collaboration, and a computer: Integrating a computer into a first-second grade writing program. Research in the Teaching of English, 20, 357378.

DiNucci, D. (1999). Fragmented future. Print, 53(4).

Dossier - Les blogues: un nouveau phénomène de société. (2005). BiblioCliQ, 3(1). Récupéré de http://www.bibliotheques.uqam.ca/informations/bibliocliq/dossiers/blogues.html

Downes, S. (2006). Learning Networks and Connective Knowledge. Instructional Technology Forum. Récupéré de http://it.coe.uga.edu/itforum/paper92/paper92.html

Engeström, Y. (1999). Activity theory and individual and social transformation. In Y. Engeström, R. Miettinen \& R.-L. Punamäki (Eds.), Perspective on activity theory: Learning in doing: social, cognitive, and computational perspectives (pp. 19-38). New York : Cambridge University Press.

Epelboin, F., \& O’Dell, J. (2009). L'usage du web social améliore la maitrise de l'écrit chez les enfants. ReadWriteWeb. Récupéré de http://fr.readwriteweb.com/2009/12/08/a-laune/lusage-du-web-social-amliore-maitrise-de-lcrit-chez-les-enfants/

Ferriter, B. (2009). Learning with blogs and wikis. Educational Leadership, 66(5), 34-38.

Freebody, P., \& Freiberg, J. (2001). Re-discovering practical reading activities in homes and schools. Journal or Research in Reading, 24(3), 222-234.

Gagnon, R., \& Ziarko, H. (2005). Analyser des textes d'élèves pour soutenir l'intervention didactique en écriture. In J. Loiselle, L. Lafortune \& N. Rousseau (Éds.), L'innovation en formation à l'enseignement (pp.123-136). Québec : Presses de l'Université du Québec.

Gaver, W. W. (1991). Technology affordances. Paper presented at the Proceedings of the SIGCHI conference on Human factors in computing systems: Reaching through technology. New Orleans, LA. 
Gibson, J. (1979). The theory of affordances. In R. Shaw \& J. Bransford (Eds.), Perceiving, Acting and Knowing. Hillsdale, NJ : Erlbaum.

Graves, D. (2003). Writing : Teachers and children at work. Portsmouth, NH : Heineman.

Greeno, J. G. (1998). The situativity of knowing, learning, and research. American Psychologist, 53(1), 5-26.

Harter, S., Whitesell-Rumbaugh, N., \& Kowalski, P. (1992). Individual differences in the effect of educational transitions on young adolescent's perceptions of competence and motivational orientation. American Educational Research Journal, 29(4), 777-807.

Hayes, J. (1995). Un nouveau modèle du processus d'écriture. In J.Y., Boyer \& J.P. Dionne (Éds.), La production de textes : vers un modèle d'enseignement de l'écriture (pp. 49-72). Montréal : Logiques.

Hayes, J. R. (2006). New directions in writing theory. In S. G. Charles, A. MacArthur, \& J. Fitzgerald (Eds.), Handbook of Writing Research (pp. 28-40). New York: Guilford.

Hayes, J., \& Flower, S. (1980). Identifying the organization of writing processes. In L.W. Gregg \& E.R. Steinberg (Eds.), Cognitive processes in writing (pp. 3-30). Hillsdale, NJ : Lawrence Erlbaum.

Hawisher, G. E. (1989). Research and recommendations for computers and composition. In G. E. Hawisher \& C. L. Selfe (Eds.), Critical perspectives on computers and composition instruction. New York: Teachers College Press.

Herrmann, A W. (1987). An ethnographic study of a high school writing class using computers: marginal, technically proficient, and productive learners. In L. Gerrard (Ed.), Writing at century's end: essays on computer-assisted composition. New York: Random House.

Hidi, S. (2001). Interest, reading, and learning : Theoretical and practical considerations. Educational Psychology Review, 13(3), 191-209.

Holdaway, D. (2000). Affinities and contradictions : The dynamics of social or learning acquisition learning. Literacy Teaching and Learning : An International Journal of Early Reading and Writing, 5(1), 7-25.

Kaplan, N. (1991). Ideology, technology, and the future of writing instruction, In G.E. Hawisher \& C.L. Selfe (Eds.), Evolving perspectives on computers and composition studies: Questions for the 1990s. Urbana, IL: National Council of Teachers of English.

Koschmann, T.D., Myers, A.C., Feltovich, P.J., \& Barrows, H. (1994). Using technology to assist in realizing effective learning and instruction: A principled approach to the use of computers in collaborative learning. The Journal of the Learning Sciences, 3, 227-264.

Kress, G. (2003). Literacy in the new media age. London: Routledge. 
Laferriere, T., Breuleux, A., \& Allaire, S. (2007). Teaching as a visible activity in remote networked schools: A socio-cultural perspective. In T. Bastiaens \& S. Carliner (Eds.), Proceedings of world conference on e-learning in corporate, government, healthcare, and Higher Education 2007 (pp. 6846-6852). Chesapeake, VA : AACE.

L’Écuyer, R. (1978). Le concept de soi. Paris: PUF.

Lee, C. K. M. (2007). Affordances and text-making practices in online instant messaging. Written Communication, 24(3), 223-249.

Levina, R. (1981). L.S. Vygotsky's ideas about the planning function of speech in children. In J. Wertsch (Ed.), The concept of activity in Soviet psychology (pp. 279-299). Armonk, NY : Sharpe.

Luckin, R., Clark, W., Graber, R., Logan, K., Mee, A., \& Martin, O. (2009). Do Web 2.0 tools really open door to learning ? Practices, perceptions and profiles of 11-16-year-old students. Learning, Media and Technology, 34(2), 87-104.

Luehmann, A., \& MacBride, R. (2008). Classroom blogging in the service of student-centered pedagogy: Two high school teachers' use of blogs. THEN: Journal, 1-23.

MacBride, R., \& Luehmann, A. L. (2008). Capitalizing on emerging technologies: A case study of classroom blogging, School Science and Mathematics, 108(5), 173-183.

Marsh, H. (1990). A multidimensional, hierarchical model of self-concept: Theoretical and empirical justification. Educational Psychology Review, 2(2), 77-172.

Means, B., Toyama,Y., Murphy. R., Bakia, M., \& Jones, K. (2009). Evaluation of evidencebased practices in online learning: A meta-analysis and review of online-learning studies. Washington, DC: U.S. Department of Education.

Ministère de l'Éducation. (2001). Programme de formation de l'école québécoise. Éducation préscolaire et enseignement primaire. Québec: Gouvernement du Québec.

Ministère de l'Éducation, du Loisir et du Sport. (2006). Programme de formation de l'école québécoise. Enseignement secondaire. Québec : Gouvernement du Québec.

Ministère de l'éducation, du Loisir et du Sport. (2008). Mieux soutenir le développement de la compétence à écrire. Rapport du comité d'experts sur l'apprentissage de l'écriture. Québec : Gouvernement du Québec.

Nadon, Y. (2007). Écrire au primaire. Réflexions et pratiques. Montréal : Chenelière éducation.

Nolen, S. B. (2007). Young children's motivation to read and write: Development in social contexts. Cognition and Instruction, 25(2), 219 - 270.

Norman, D. (1983). Design principles for human-computer interfaces. ACM CHI 83 Human Factors in Computing Systems Conference, Boston.

O’Reilly, T. (2004). Open source paradigm shift. Récupéré de http://tim.oreilly.com/articles/paradigmshift_0504.html 
Passey, D. (2001). Anytime anywhere learning pilot programme: A Microsoft UK supported laptop project : Learning gains in Year 5 and Year 8 classrooms. Reading : Microsoft.

Pearson, D., \& Taylor, B. (1994). What really matters : The impact of schoolwide and classroom practices on growth in students' reading achievement. International Reading Association, Reno.

Pintrich, P., \& Schunk D. (1996). Motivation in education: Theory, research, and applications. Englewood Cliffs, NJ : Merrill/Prentice Hall.

Powell, S., \& Nelson, B. (1997). Effects of choosing academic assignments on a student with attention deficit hyperactivity disorder. Journal of Applied Behavior Analysis, 30(1), 181183.

Préfontaine, C. (1998). Écrire et enseigner l'écriture. Montréal : Éditions Logiques.

Prensky, M. (2001). Digital Natives, Digital Immigrants. On The Horizon, 9(5). Récupéré de http://www.marcprensky.com/writing/Prensky\%20-

\%20Digital\%20Natives,\%20Digital\%20Immigrants\%20-\%20Part1.pdf

Ramswami, R. (2008). The Pros (and a few cons, too) of blogging. T.H.E. Journal, 35(11), 2125.

Rijlaarsdam, G., Braaksma, M., Couzijn, M., Janssen, T., Raedts, M., van Steendam, E., Toorenaar, A., \& van den Bergh, H. (2008). Observation of peers in learning to write. Journal of writing research, 1(1), 53-83.

Ross, J. (1973). A study of the effect of the learning environment on selected factors related to the self-concept of school children. Dissertation abstracts international, 33(12-A), 6600.

Ross, J. A. (1988). Controlling variables: A meta-analysis of training studies. Review of Educational Research, 58(4), 405-437.

Routman, R. (2010). Enseigner l'écriture : revenir à l'essentiel. Montréal : Chenelière éducation.

Roy, R. (2009). Enquête sur la génération C. Les 12-24 ans : utilisateurs extrêmes d'Internet et des TI. Montréal: CEFRIO.

Ryan, R. M., \& Deci, E. L. (2000). Intrinsic and extrinsic motivations: Classic definitions and new directions. Contemporary Educational Psychology, 25(1), 54-67.

Schiefele, U. (2001). The role of interest in motivation and learning. In S. Messick \& J. M. Collis (Eds.), Intelligence and personality. Hillsdale, NJ: Erlbaum.

Sharples, M., Graber, R., Harrison, C., \& Logan, K. (2009). E-Safety and Web 2.0 for children aged 11-16. Journal of Computer Assisted Learning, 25(1), 70-84.

Scrimshaw, P. (1997). Computers and the teacher's role. London : Routledge. 
Simard, C. (1995). Éveil du goût d'écrire. In St-Laurent, L., Giasson, J., Simard, C., Dionne, J.J., Royer, É. et coll. Programme d'intervention auprès des élèves à risque. Une nouvelle option éducative (pp.145-149). Boucherville, QC : Gaëtan Morin.

Snow, C., Burns, S., \& Griffin, P. (1998). Preventing reading difficulties in young children. Washington : National Academy Press.

Snyder, I. A. (1993). The impact of computers on students' writing: A comparative study of the effects of pens and word processors on writing context, process and product. Australian Journal of Education, 37, 5-25.

Snyder, I. A. (1994). Writing with word processors: The computer's influence on a classroom context. Journal of Curriculum Studies, 26, 143-62.

Sousa, S., \& Soares M. (2009). Developing writing skills through the use of blogs. L1 Educational Studies in Language and Literature, 9(2), 71-90.

Thomas, J. (1980). The self in education. Windsor, UK : NFER Publishing Co.

Tomé, M. (2009). Productions orales, weblogs et projet de télécollaboration avec le web 2.0 pour l'enseignement du français (FLE). Alsic, 12. Récupéré de http://alsic.revues.org/index1279.html

Turcotte, C. (2007). Engager l'élève du primaire en lecture. Montréal : Chenelière éducation.

Viau, R. (1994). La motivation en contexte scolaire. St-Laurent, QC : Éditions du Renouveau pédagogique.

Viau, R. (1999). La motivation dans l'apprentissage du français. St-Laurent, QC : Éditions du Renouveau pédagogique.

Vygotsky, L. (1978). Mind in society : The development of Higher psychologial processes. Cambridge, MA : Harvard University Press.

Ward, J. (2004). Blog assisted language learning (BALL): Push button publishing for the pupils. TEFL Web Journal, 3(1).

Watson, D. (1993). The ImpacT Report: An evaluation of the impact of information technology on children's achievements in primary and secondary schools. London: Department for Education and King's College Centre for Educational Studies, London.

West, K. (2008). Weblogs and literary response : Socially situated identities and hybrid social languages in English class blogs. Journal Of Adolescent \& Adult Literacy, 51(7), 588-598. 


\section{Auteurs}

Stéphane Allaire est professeur en pratiques éducatives à 1'Université du Québec à Chicoutimi. Courriel : Stephane_Allaire@uqac.ca.

Pascale Thériault est professeure en apprentissage de la lecture et de l'écriture à l'Université du Québec à Chicoutimi.

Evelyne Lalancette est étudiante à la maîtrise en éducation à l’Université du Québec à Chicoutimi. 